\title{
Acoustic Parameters as Criteria of Localized Deformation in Aluminum Alloys
}

\author{
A.G. Lunev*, M.V. Nadezhkin, S.A. Barannikova And L.B. Zuev \\ Institute of Strength Physics and Materials Science, Siberian Branch, Russian Academy of Sciences, \\ 634055 Tomsk, Russia
}

\begin{abstract}
Investigations of propagation velocity and attenuation change of the Rayleigh waves depending on plastic strain localization in materials having the Portevin-Le Chatelier effect are presented in this paper. Measurements of the ultrasound wave characteristics were carried out in situ during specimen tensile at the constant rate and room temperature. Registration of localized strain band kinetics was performed with the digital speckle photography method. The regularity of ultrasound velocity change during the band propagation through the ultrasound measurement area was discovered.
\end{abstract}

DOI: 10.12693/APhysPolA.134.342

PACS/topics: Portevin-Le Chatelier effect, jerky flow phenomenon, ultrasound velocity, ultrasound attenuation, localized strain

\section{Introduction}

The jerky flow phenomenon, called the Portevin-Le Chatelier (PLC) effect, is one of the most investigated problems of plastic deformation. However, experimental and theoretical researches of this phenomenon are continuing nowadays. From a dynamical point of view, jerky flow is discontinuous dislocation motion limited by pinning and unpinning of dislocations. Widely used explanation of PLC effect is based on the dynamic strain ageing (DSA) concept. This concept was firstly introduced by Cottrell [1] and then expanded by McCormick [2], Penning [3], Kubin et al. [4] and other. DSA manifests due to the diffusion of solute atoms to the temporary arrested mobile dislocations by volume or pipe diffusion. Solute atoms pin the dislocations. The longer the dislocations (or the slower the strain) the larger stress required to unpin them. At the large deformation rate the solute atoms do not have enough time to diffuse to the mobile dislocations. Therefore, hindering of dislocations is limited by interaction with dislocation forest and other lattice defects in metal. In this case, stress-stain diagram is monotonic. The same monotonic plastic flow curve can be obtained in the case of low strain rate and simultaneously high speed of the solute atom diffusion. The atoms pin temporary arrested at obstacles dislocations, therefore it requires large stress for dislocation unpinning.

A PLC band generation is the result of the dislocation assembly motion and dislocation forest formation. Some researchers suggest estimating the mobile dislocation density by the measurement of acoustic emission (AE) parameters. For example in [5-8], it has been found that PLC band nucleation is accompanied by a high level acoustic emission. The number of dislocations involved

*corresponding author; e-mail: agl@ispms.tsc.ru in PLC band generation is order of $10^{4}$. The evaluation of dislocation assembly scope propagating in the form of PLC bands and their location along tested specimen can be carried out by the measurement of the velocity and the attenuation of ultrasound waves $[9,10]$. Theoretical ground of dislocation influence on the ultrasound velocity and attenuation was firstly presented by Granato and Lücke $[11,12]$ as part of the internal friction theory. Later this theory was expanded by Maurel [13]. Experimental investigations of the propagation velocity and the attenuation of ultrasound waves in metallic materials during plastic flow were carried out by Gremaud et al. [14], by Ogi et al. [15], by Zuev et al. [16, 17] and Kobayashi [18, 19].

In the present paper the ultrasound velocity variation in aluminum alloys during plastic flow is under investigation. There was studied the possibility of definition of PLC band location and level of local deformation in the band by the ultrasound parameter measurement.

\section{Materials and methods}

The investigations were performed for $\mathrm{AlCu} 4 \mathrm{MgSi}$ and AlMg5 alloys. Plane samples were made in a dog-bone shape, with working part dimensions $50 \times 10 \times 2 \mathrm{~mm}^{3}$. After manufacturing the samples were annealed during $3 \mathrm{~h}$ at the temperature of $633 \mathrm{~K}$ for $\mathrm{AlCu} 4 \mathrm{MgSi}$ and $763 \mathrm{~K}$ for AlMg5 alloys for residual stress removal.

Samples were tensile tested with the LFM-125 testing machine at a constant strain rate of $0.2 \mathrm{~mm} / \mathrm{min}$ at the room temperature.

Investigations of plastic deformation localization were performed during the strain using two methods - DIC on the automated laser measuring complex ALMECtv [20] and ultrasound parameters measurement.

The DIC method involves processing of speckle pattern sample's deformed surface, illuminated with a coherent laser beam. As a result we obtain the digital image sequence of a sample observed part with rate up to 
50 frames per second. Following frame digital treatment provides estimating of $U$ and $V$ vector fields, from which are possible to obtain longitudinal $\left(\varepsilon_{x x}\right)$, transverse $\left(\varepsilon_{y y}\right)$, shear $\left(\varepsilon_{x y}\right)$ and rotational $\left(\omega_{z}\right)$ components of the plastic distortion tensor.

Piezoelectric emitter of ultrasound (Rayleigh) waves and receiving transducer were made in one sensor body. Bases of measurements (distance between emitter and receiver) for used sensors were 5 and $32 \mathrm{~mm}$. An electric pulse from generator excited the emitting transducer with duration $100 \mathrm{~ns}$ and repeat time $30 \mu \mathrm{s}$. Acoustic wave propagation velocity was measured as relation between path length and the delay time of the pulse. Relative ultrasound wave velocity was calculated as $\nu=$ $V_{R} / V_{R 0}$, where $V_{R}$ is the current wave velocity, $V_{R 0}$ is the wave velocity in initial condition. The error of this ultrasound method is $\pm 0.1 \mathrm{~m} / \mathrm{s}[21]$.

\section{Results and discussions}

The investigations of velocity and attenuation of the Rayleigh waves on $\mathrm{AlCu} 4 \mathrm{MgSi}$ alloy were performed by the sensor with $32 \mathrm{~mm}$ base (large base). The sensor was located on a wide working side of the sample, opposite side was illuminated by scattered laser light. Speckle patterns arisen on the surface were recorded with a high resolution digital camera. On the all of the samples, the parabolic stage of plastic yielding was established. The strain hardening exponent on this stage is $n=1 / 2$. Then the prefracture stage is following, which is ending by deformation localization in position corresponding to the neck of fracture. The AlCu4MgSi alloy is precipitation hardening alloy deformed with PLC effect appearance. This effect has been expressed in stress surges (Fig. 1a).

It was found that the falls more than $6 \mathrm{MPa}$ occurred by the nucleation of localized strain bands. These bands propagate along the specimen. Registration of bands on the working specimen surface by digital speckle images treatment was performed. The decrease in bands velocity from $1.0 \mathrm{~mm} / \mathrm{s}$ to $0.3 \mathrm{~mm} / \mathrm{s}$ with the strain increase was established. This effect is pointed out in many studies of PLC effect $[22,23]$. In contrast to hyperbolical dependence of localization velocity versus total deformation used in literature, we suggested a linear approximation of bands velocity versus strain hardening exponent.

The tendency to decrease in the Rayleigh waves velocity propagation with the strain increase was observed. However, this dependence is nonmonotonic (Fig. 1b). The height and length of the peaks enhance with the total strain increase.

The results were obtained by the optical method and by the measurement of ultrasound velocity were matched. The decrease in localized strain band velocity over the range of ultrasound measurements (length between transducers) was determined, while the band propagation outside the measurement area led to ultrasound velocity increase.

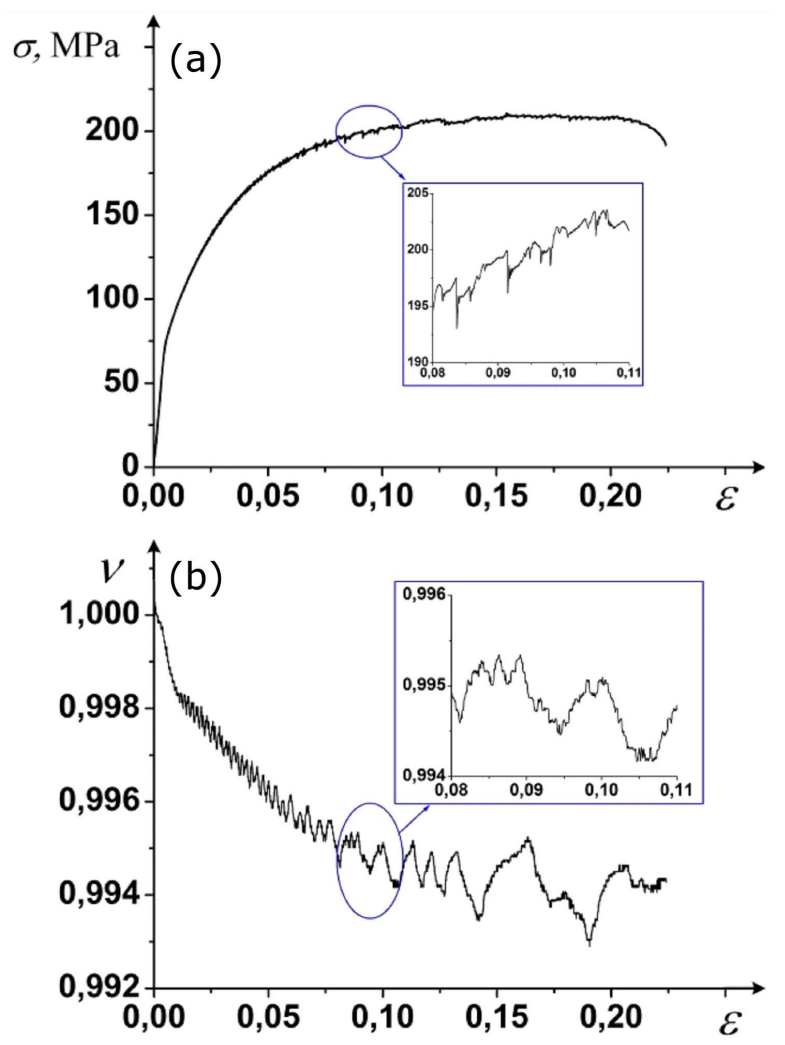

Fig. 1. Stress (a) and Rayleigh waves velocity (b) versus deformation of $\mathrm{AlCu} 4 \mathrm{MgSi}$ alloy plot.

The ultrasound velocity dependence on the place of local straining zones using the sensor with the base length equal to $5 \mathrm{~mm}$ (short base) was established. Short base allows defining the influence of the band passing more accurately. It allows perform evaluated calculations. The study was performed on the AlMg5 alloy specimens showing the intense PLC effect during plastic deformation.

It was established that AlMg5 alloy deformation includes parabolic and prefracture stages. The same stages were seen in $\mathrm{AlCu} 4 \mathrm{MgSi}$ alloy. The duration of parabolic stage is $15 \%$ of total deformation, prefracture is $7 \%$, and then the fracture neck appears. It was established that the stress dump and PLC band nucleation coincide. Stress drop value on the stress-strain diagram rise with strain rising from $6 \mathrm{MPa}$ to $24 \mathrm{MPa}$. However, in spite of jerky movement of bands, deformation covers the whole sample.

The tendency for nucleation or propagation PLC bands depending on the fracture position was not revealed. The nucleation of fracture neck happened in random places and it was unpredictable. Changes in the Rayleigh wave velocity confirmed the impact of the strain localization on ultrasound waves. The results of the comparison of places of PLC band with the Rayleigh wave velocity changes are shown in Fig. 2a and b. The Rayleigh wave attenuation changes in opposite phase with velocity. In other words - localized strain band movement leads to attenuation increase, while ultrasound velocity decreases. 
It was noted that PLC band motion through the ultarasound measurement area leads to ultrasound velocity decrease. The magnitude of this decrease depends on the duration of the band occurrence between the ultrasound transducers as well as the local strain rate in band. The strain rate in the bands was measured with DIC method. This required $\varepsilon_{x x}$ (specimen elongation) deformation component measurement over a certain time interval $\Delta t$. In this study $\Delta t$ ranged from 2 to $6 \mathrm{~s}$. The investigations have shown the proportional decrease of the Rayleigh wave amplitude with the total strain. This decrease is caused by PLC band propagation within acoustic measurements area.

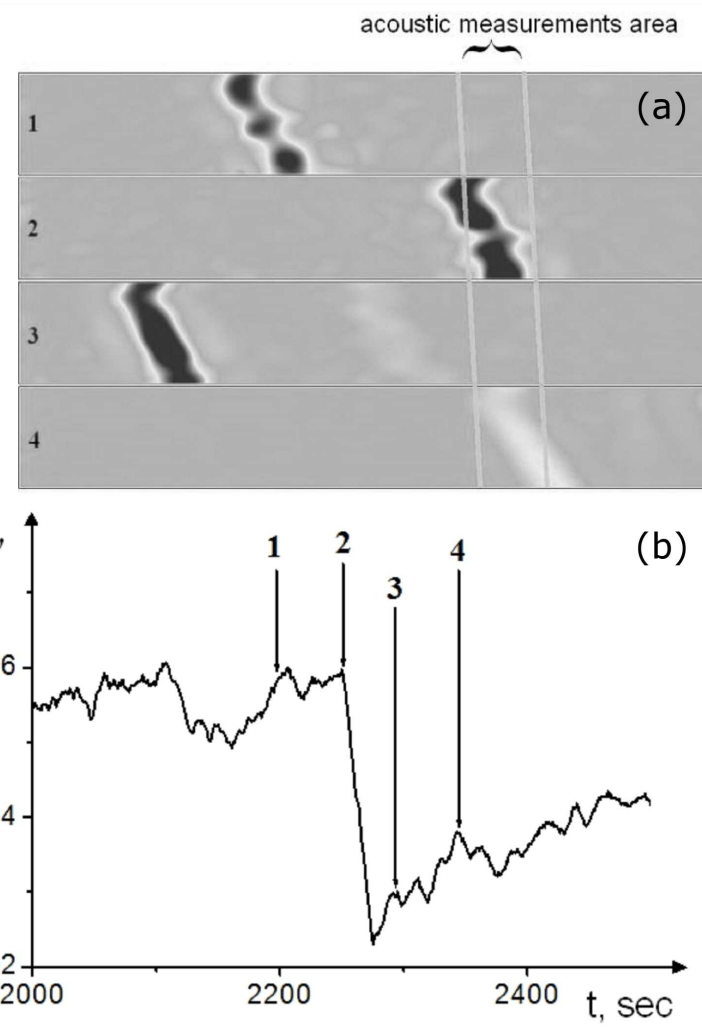

Fig. 2. Localized strain locations in AlMg5 alloy concerning the acoustic measurements area (a). Impact of localized strain on ultrasound velocity in AlMg5 alloy sample (b). Moments of strain localization registration are marked by numbers.

DIC method provided the possibility to identify the change of band velocity according to the total specimen strain. It was revealed that the deformation velocity in PLC band was increasing simultaneously with the total strain. Character of enhancing of strain rate is close to logarithmic dependence. According to the Orovan equation the deformation velocity can be written via dislocation parameters as

$$
\dot{\varepsilon}=b \rho_{m} v_{d},
$$

where $b$ is the length of the Burgers vector, $\rho_{m}$ is the mobile dislocations density, $v_{d}$ is the mobile dislocations velocity.
Assuming the mobile dislocations density stationary (bands width is not changing), the proportional correlation between the strain rate in bands and dislocation velocity was obtained. The fact that dislocation velocity is increasing exponentially with the increase of the difference between internal and external stress supports this idea [24].

\section{Conclusion}

The experiments on $\mathrm{AlCu} 4 \mathrm{MgSi}$ and $\mathrm{AlMg} 5$ alloys were performed. These materials manifest PLC effect in the process of deformation. Simultaneous registration of the sample surface change with DIC method and the acoustic parameters measurements provided the possibility to point that the deformation velocity in localized band increases with stress increase. This conclusion supports the Orovan equation and McCormic ideas of the applied stress influence on dislocation velocity. Changes in the Rayleigh wave velocity correspond to deformation magnitude, which occurred in ultrasound controlled area of the sample. Obviously, ultrasound wave velocity change can be criteria of localized deformation nucleation during the pressure treatment of aluminum alloys.

\section{Acknowledgments}

The work was supported by the Russian Science Foundation (project No. 16-19-10025).

\section{References}

[1] A.H. Cottrell, Philos. Mag. J. Sci. 44, 829 (1953).

[2] P.G. McCormick, Acta Metall. 20, 351 (1972).

[3] P. Penning, Acta Metall. 20, 1169 (1972).

[4] L.P. Kubin, K. Chihab, Y. Estrin, Acta Metall. 36, 2707 (1988).

[5] H. Louche, K. Bouabdallah, P. Vacher, T. Coudert, P. Balland, Exp. Mech. 48, 741 (2008).

[6] F. Chmelík, A. Ziegenbein, H. Neuhäuser, P. Lukáč, Mater. Sci. Eng. A 324, 200 (2002).

[7] C.H. Cáceres, A.H. Rodriguez, Acta Metall. 35, 2851 (1987).

[8] I.V. Shashkov, M.A. Lebyodkin, T.A. Lebedkina, Acta Mater. 60, 6842 (2012).

[9] N. Mujica, M.T. Cerda, R. Espinoza, J. Lisoni, F. Lund, Acta Mater. 60, 5828 (2012).

[10] F. Barra, R. Espinoza-González, H. Fernández, F. Lund, A. Maurel, V. Pagneux, JOM 67, 1856 (2015).

[11] A. Granato, K. Lücke, J. Appl. Phys. 27, 583 (1956).

[12] A. Granato, K. Lücke, J. Appl. Phys. 27, 789 (1956).

[13] A. Maurel, V. Pagneux, F. Barra, F. Lund, Int. J. Bifurc. Chaos 19, 2765 (2009).

[14] G. Gremaud, M. Bujard, W. Benoit, J. Appl. Phys. 61, 1795 (1987)

[15] H. Ogi, N. Suzuki, M. Hirao, Metall. Mater. Trans. A 29, 2987 (1998).

[16] L.B. Zuev, B.S. Semukhin, Philos. Mag. A 82, 1183 (2002). 
[17] L.B. Zuev, L.B. Semukhin, A.G. Lunev, Meas. Tech. 53, 439 (2010).

[18] M. Kobayashi, Int. J. Plast. 14, 511 (1998).

[19] M. Kobayashi, Int. J. Plast. 14, 523 (1998).

[20] L.B. Zuev, V.V. Gorbatenko, K.V. Pavlichev, Meas. Sci. Tech. 21, 1 (2010).

[21] A.G. Lunev, M.V. Nadezhkin, L.B. Zuev, in: Int. Conf. on Advanced Materials with Hierarchical Structure for New Technologies and Reliable Structures 2016, AIP Conf. Proc., Vol. 1683, Ed. V.E. Panin et al., American Institute of Physics, Melville (NY) 2016, p. 020140-1.
[22] N. Ranc, D. Wagner, Mater. Sci. Eng. A 474, 188 (2008).

[23] A. Benallal, T. Berstad, T. Borvik, O.S. Hopperstad, I. Koutiri, R. Nogueira de Codes, Int. J. Past. 4, 1916 (2008).

[24] P.G. McCormick, Y. Estrin, Scr. Metall. 23, 1231 (1989). 